\title{
LYCORIS: A large area beam telescope based on hybrid-less strip silicon sensors
}

\author{
Mengqing Wu, Martin Breidenbach, Dietrich R. Freytag, Uwe Kraemer, \\ Benjamin A. Reese, Sebastiaan Roelofs and Marcel Stanitzki
}

\begin{abstract}
A new Large area $x-Y$ COverage Readout Integrated Strip telescope (LYCORIS) is being constructed as an improvement of the DESY test beam infrastructure within the Horizon2020 AIDA-2020 project [1]. The LYCORIS telescope consists of six layers of $25 \mu \mathrm{m}$ pitch strip Si sensor readout by two bump-bonded ASICs (KPiX), running at a timing resolution as multiples of $80 \mathrm{~ns}$; its active area is designed to be $10 \times 10 \mathrm{~cm}^{2}$, extendable to $10 \times 20 \mathrm{~cm}^{2}$. It can run either standalone or be mounted inside a $1 \mathrm{~T}$ solenoid magnet, providing a spatial resolution better than $10 \mu \mathrm{m}$ along the bending direction, and a resolution better than $1 \mathrm{~mm}$ along the magnetic field. The full readout system was tested with a hexagonal pixel sensor designed for the SiD ECAL in the lab with a ${ }^{90} \mathrm{Sr}$ source, and later tested in the electron beam at DESY in May and October 2017. The first assembled modules with the final strip were tested in spring 2018. First results of the LYCORIS prototype will be presented with a comparison to simulation, besides, the characterization of sensor and readout system are also included.
\end{abstract}

\section{INTRODUCTION}

The DESY II Test Beam Facility [2] provides $e^{-} / e^{+}$ beams with energies from 1 to $6 \mathrm{GeV}$ up to $1 \mathrm{MHz}$ with three test beamlines (TB21, TB22 and TB24). The beamlines TB21 and TB22 are both equipped with a EUDET-type beam telescope [4] with an active area of around $1 \times 2 \mathrm{~cm}^{2}$, which is based on a fine pitch MIMOSA 26 monolithic sensor with an event readout frame of $115.2 \mu \mathrm{s}$; the beamline TB24 is equipped with a $1 \mathrm{~T}$ solenoid, the PCMAG, at a diametre of $\sim 85 \mathrm{~cm}$ with its wall of $\sim 20 \mathrm{X}_{0}$, inside which both the EUDET-type telescope and the Device Under Test (DUT) can be inserted. The EUDET-type beam telescopes at DESY are proven to meet user demands very well and thus in a high $(\sim 80 \%)$ demand, however, its active area is limited for momentum measurements for users in the PCMAG or for users requiring a larger tracker coverage. Therefore, a new telescope is in need to provide a spatial resolution better than $10 \mu \mathrm{m}$ along the bending direction, and a resolution better than $1 \mathrm{~mm}$ along the magnetic field, with a large

Manuscript received December 14, 2018; revised January 9, 2019. This project has received funding from the European Union's Horizon 2020 Research and Innovation programme under Grant Agreement no. 654168.

M. Wu, U. Kraemer and M. Stanitzki are with Deutsches ElektronenSynchrotron DESY, Germany (email: mengqing.wu@desy.de).

M. Breidenbach, D. R. Freytag and B. A. Reese are with Stanford Linear Accelerator Center SLAC, USA.

S. Roelofs is with The Hague University of Applied Sciences, The Netherlands, and was with Deutsche Elektronen-Synchrotron DESY, Germany.

(C) All figures and pictures by the author(s) under a CC BY 4.0 license (see https://creativecommons.org/licenses/by/4.0). active area of $10 \times 10 / 20 \mathrm{~cm}^{2}$, to cover $90 \%$ to $96 \%$ of the incoming particles with energies from 1 to $6 \mathrm{GeV}$ considering momentum smearing after the magnet wall.

The $320 \mu \mathrm{m}$ thick, $10 \mathrm{~cm}$ long, $10 \mathrm{~cm}$ wide SiD microstrip sensor has a sense/readout pitch of $25 / 50 \mu \mathrm{m}$, which can provide a resolution of around $7.2 \mu \mathrm{m}$, and hence it was chosen. The other requirements can be achieved by a $2^{\circ}$ tilt. This sensor was originally developed for the SiD Detector Concept for the ILC [6].

LYCORIS is designed as a 6-plane strip telescope, each of which consists of two sensors side-by-side along the axis of the magnetic field. The sensor plane orientation is flexible according to the user demands, however, a default design is organized to cope with a large user device in the PCMAG, as following.

- The upstream arm and the downstream arm are organized mirror symmetric, where upstream arm refers to the first three planes that the incident particle passes, hence downstream arm refers to the last three planes.

- The upstream sensors are organized with $\left(0,-2^{\circ}, 2^{\circ}\right)$ stereo angles to achieve the requested spatial resolution with a distance of $15 \mathrm{~cm}$ between sensors.

- Each half is packed in a cassette-like Aluminium box, see in Fig. 1, which is covered by two Carbon fibre plates, and with two little boards installed at the bottom of the cassette, for power distribution and data transmission.

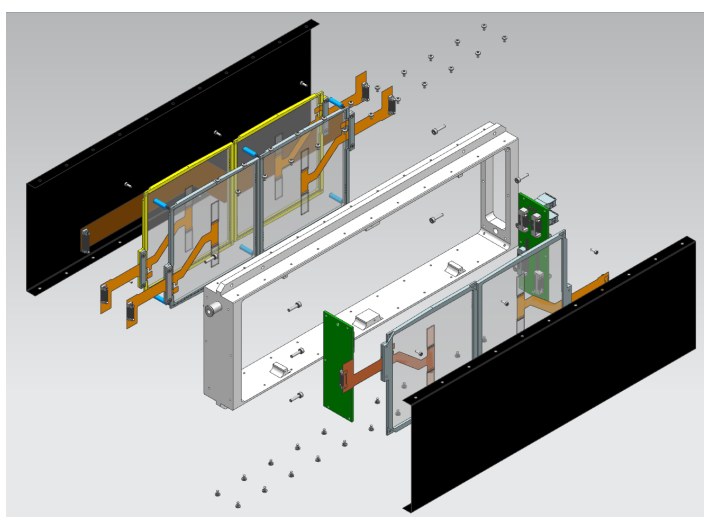

Fig. 1. CAD drawing of each component of one half of the telescope, i.e. three layers of two microstrip sensors in parallel, installed in a cassette-like support with two local readout electronic support at the endplate.

The signal is sent out from the on-sensor ASIC to the FPGA-based DAQ board, via a Kapton flex and the small 


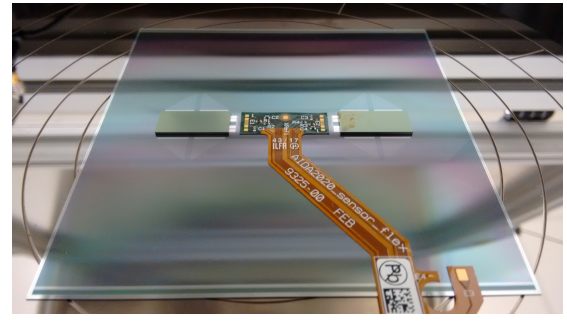

Fig. 2. Photo of an assembled module with two bump-bonded KPiX chips. A kpaton flex cable is glued on the sensor, and communicate with the KPiX through a second metallization layer.

board at the bottom of the cassette. The DAQ board is capable to receive a coincidence from a connected Trigger Logic Unit (TLU) [3], timestamp it and use it as an external trigger.

\section{A. SiD Hybrid-less microstrip Sensor}

The SiD strip sensor is readout by a bump-bonded 1024channel ASIC, KPiX [5], through a second metallization layer. The KPiX chip digitizes the signal with a 13 bit ADC resolution, then send out through the second metallization layer to a wirebonded Kapton cable, see Fig. 2. This hybridless organizing eliminates the need for a complex hybrid design, and its functioning is first-time tested in the LYCORIS project.

The KPiX chip runs in a pulse cycle mode, i.e. the chip will receive a power-on pulse to be active for a certain period of time, then power cycled. This cycle, later noted as acquisition cycle, is configurable based on a $100 \mathrm{MHz}$ clock, and during which each readout channel can store up to four events. The KPiX readout system was characterized before the strip sensor produced with several hexagonal pixel sensors, which were originally designed for the SiD ECAL [6]. This ECAL sensor was later used as a reference device in the test beam for the strip sensor.

The strip sensors were delivered by Hamamatsu in summer 2017, showing very good electric features and all fully depleted at around $50 \mathrm{~V}$, see Figure 3. The first assembled sensors were produced in early spring 2018, then examined firstly in the lab, then moved to the DESY II Testbream Facility for testing in summer 2018.

\section{LAB TEST}

The test setup holding structure is grounded as a Faraday shield to provide a relatively noise-free environment. Various data taking mode were tested in the lab, including ADC calibration, forced trigger pedestal measurement, self-trigger mode run with background noise and with a signal.

The calibration is done via injecting a known charge to each channel and checking its ADC response, see the ADC response to the injected charge (fC) for one readout channel in Fig. 4, a slope to convert ADC to $\mathrm{fC}$ can be determined from the linear fit performed on this calibration distribution for every channel. The calibrated slopes of all the readout channels from one $\mathrm{KPiX}$, based on the measurements in the lab, is shown by the blue histogram in Fig. 5 (left). One can determine the intrinsic

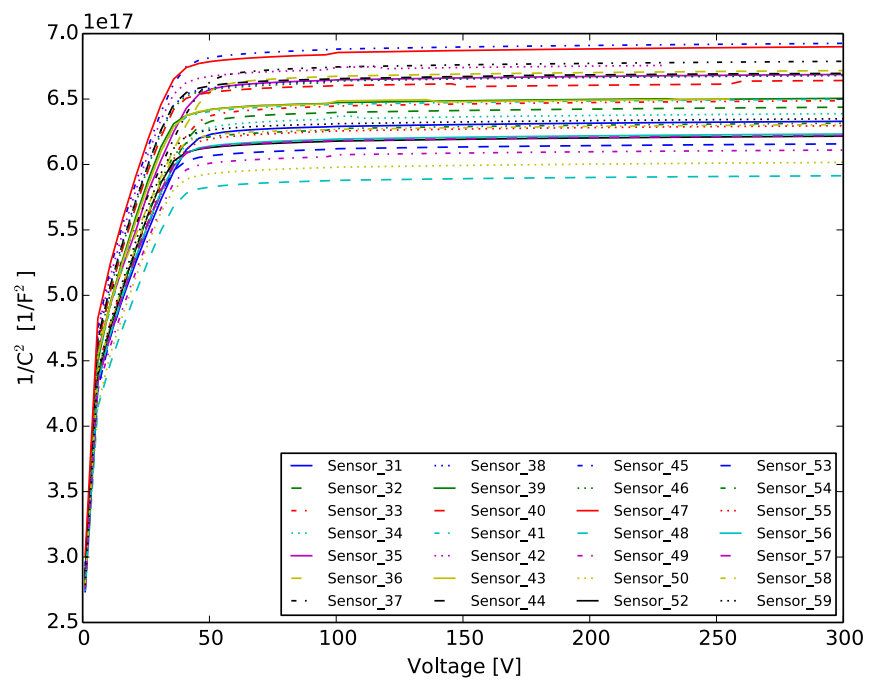

Fig. 3. C-V curves measured on all the bare sensors delivered from Hamamatsu in summer 2017, indicating all the sensors starts to be fully depleted, at around $50 \mathrm{~V}$.

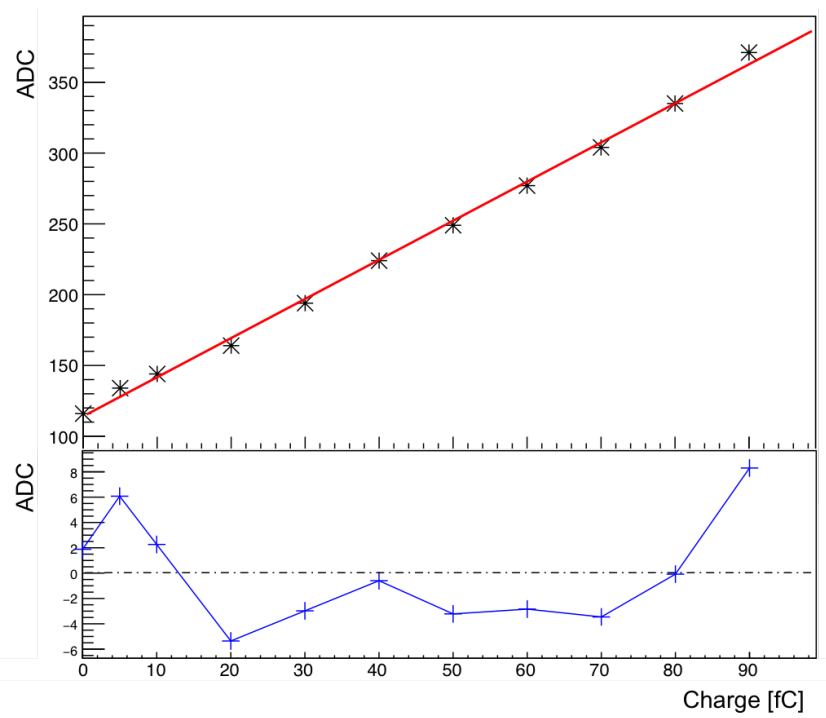

Fig. 4. Calibration of one channel, showing good ADC response to Charge (fC) change; the black dots from the upper plot shows the five DAC/fC calibration points/steps, and a linear fit was performed shown by the red line; a corresponding residual plot from the data to the fit is shown in the lower pad.

noise level of each channel, by recording random noise with a forced trigger configuration. A preliminary average noise level of $\sim 0.5 \mathrm{fC}$ is determined from the distribution of RMS of all the channels, see the blue histogram in Fig. 5 (right).

The KPiX chip is capable to disable every single channel under the self-trigger mode. Therefore, bad channels, such as channels with slopes at 0 , and with pedestal RMS values far from the average value, are disabled. Various run configurations were studied with the self-trigger mode, for example, a fine stepped threshold scan was performed, in order to choose a threshold which suppresses largely the noise. A ${ }^{90} \mathrm{Sr}$ source was used as the signal, pointing at the sensor with a collimator. The sensor shows the location of the signal position, see the 

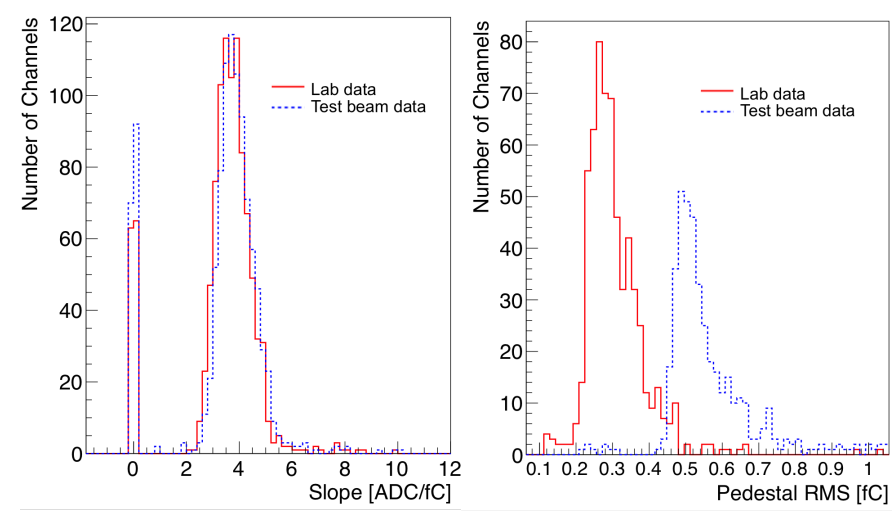

Fig. 5. The blue histogram refers to the lab measurements, while the red refers to the test beam results. Left: Slopes of ADC/fC determined from the calibration run for all the channels; Right: RMS in $\mathrm{fC}$ of pedestal measured in forced trigger run from all channels.

lit-up strips in Fig. 6, using data taken in the self-trigger mode with a tight threshold.

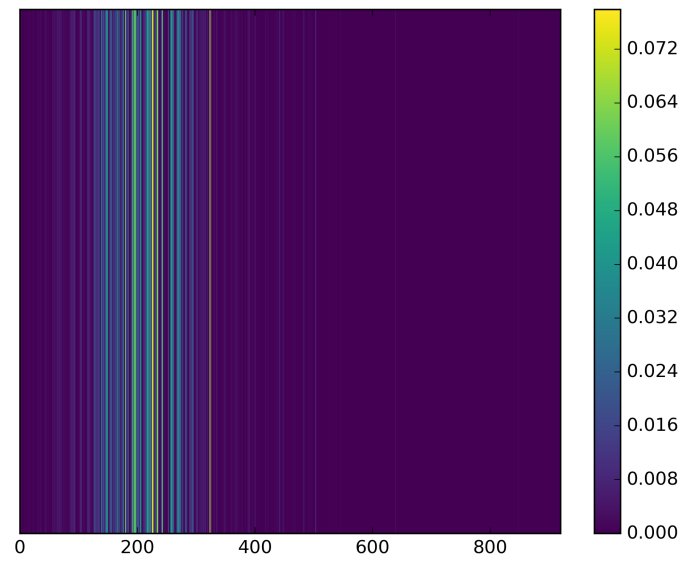

Fig. 6. Signal response plot of one KPiX, i.e. one half of the sensor, from self-trigger data taken in the lab with tight threshold, that $\mathrm{x}$-axis illustrates the strip number, while z-axis shows how likely this strip can register one event per acquisition cycle. The blacked-out strips in the signal region are the bad channels that are manually disabled according to the calibration and the pedestal behaviour.

The signal test with the sensor in the lab is only studied under the self-trigger mode, because the source energy is not high enough to pass through an external trigger device, i.e. a scintillator. One can also install the external trigger behind the sensor, however, it is found from the previous studies that the signal strength is largely decreased after passing one sensor layer.

\section{Test BeAm}

The DESY II Test Beam Area T24/1 and its infrastructure were used [2]. The beam energy was chosen to be $3 \mathrm{GeV}$ to achieve the best beam rate, and a $9 \times 9 \mathrm{~mm}^{2}$ secondary collimator was chosen. The trigger rate turns out to be $5 \mathrm{kHz}$ during the data taking period of $\mathrm{KPiX}$. The test setup at the test beam is similar to the one in the lab, but the following three changes were applied.

- Sensors were held in the final cassette support which provides better shielding, see Fig. 7.

- A hexagonal pixel sensor plane was placed behind the microstrip sensor as a reference device, which employs the same KPiX readout system.

- Two scintillators installed right after the secondary collimator, before the microstrip sensor, which were connected to a series of NIM modules to provide a coincidence to the KPiX readout system.

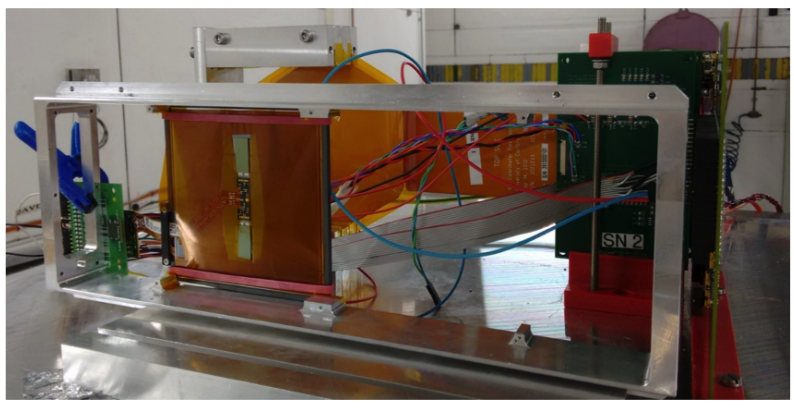

Fig. 7. Experimental setup at the DESY II Test Beam Area T24/1.

All the readout channels were re-calibrated due to the changes in the experimental setup and environment. The slopes, see the red histogram in Fig. 5 (left), stay the same as the lab measurement, which is expected. The noise level turns to be lowered to $\sim 0.2 \mathrm{fC}$, see the red histogram in Fig. 5 (right), which is expected for a better shielding environment from the grounded cassette.

\section{Preliminary Analyses and Discussions}

The same idea, as the measurement in the lab to check signal response with a tight threshold in self-trigger mode, is also implemented. The sensor shows a good capability to locate the beam position, and the size, see Fig. 8.

The self-trigger timing feature of the reference ECAL sensor was studied before [7], while this is the first time to study it on this microstrip sensor. The difference between the self-trigger time stamps and the external trigger time stamps are plotted in Fig. 9. A good agreement from the strip sensor to the reference sensor is shown on the peak at $\Delta T=1$, while the two small peaks are found every minimum trigger time unit ${ }^{1}$ after the peat at 1 , only from the strip sensor. These two peaks are believed to be events retriggered from the neighbour channels due to the large current induced during the trigger reset.

The above timing study makes it possible to suppress pedestal noise, as well as the retrigger events, by matching the self-triggered events to the external trigger timestamps, which will later on called time correlated events. It is demonstrated from the example ADC distribution recorded from one strip in the beam shown in Fig. 10 (upper), that the distribution of the time correlated events (red histogram) very well replicate the tail of the distribution of all the self-triggered events (blue

\footnotetext{
${ }^{1}$ The minimum trigger time unit is the reset time after a trigger, that was set as 8 times the BunchClockCount, a count based on the data taking clock.
} 


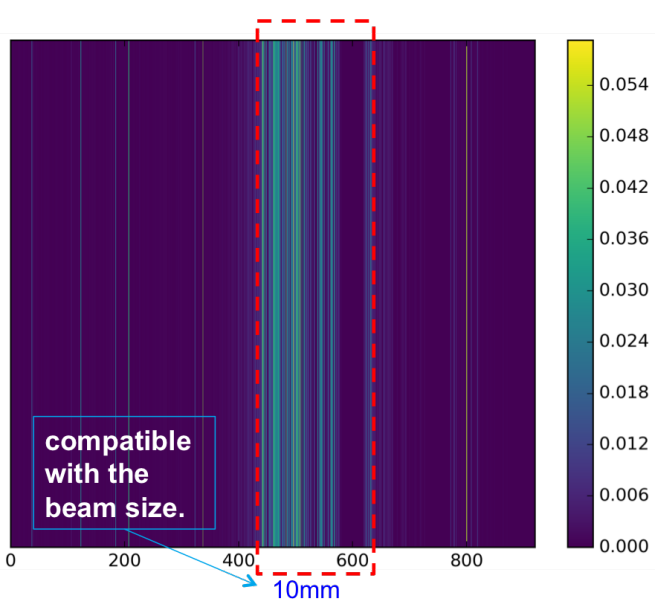

Fig. 8. Signal response plot of one KPiX, i.e. one half of the sensor, from selftrigger data taken in the Test Beam with tight threshold, that $\mathrm{X}$-axis illustrates the strip number, while z-axis shows how likely this strip can register one event per acquisition cycle. The signal region, indicated by the dashed red line, is $10 \mathrm{~mm}$ width, which is compatible with the beam size. The blackedout strips in the signal region are the bad channels that are manually disabled.

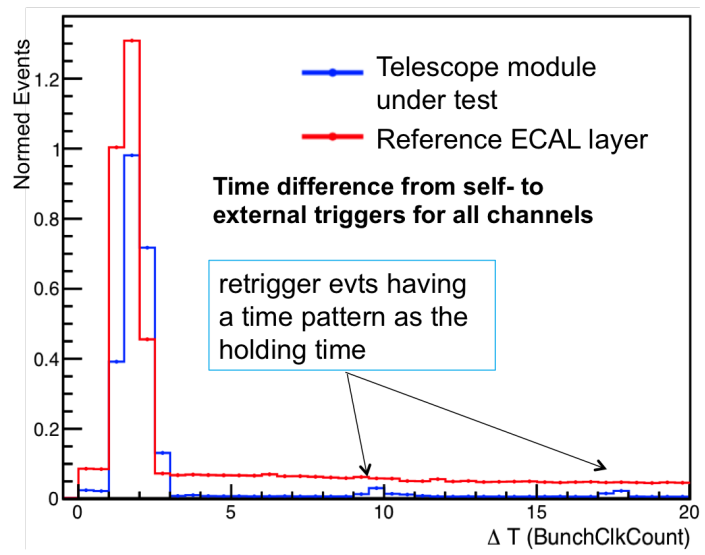

Fig. 9. Distribution of time difference $\Delta T$ from self-triggered events to the external triggers, in BunchClockCount (a count based on the data taking clock); all events from all the channels are plotted.

histogram); moreover, a reasonably good Landau fit can be performed on the time correlated distribution. This external trigger timestamp matching criterion helps to study the purity of the self-triggered events against various thresholds. Fig. 10 (lower) shows ADC distribution of a different strip in beam area from data with a tighter threshold; all the events can be matched to the external trigger in timing, and a Landau convoluted Gaussian fit can be performed which represents well the data distribution.

All the results shown here are performed without pedestal subtraction, due to a known issue of the pedestal shifting between acquisition cycles. Further analyses have been carried out after this presentation to study methods to compensate this shifting for pedestal subtraction.

\section{CONCLUSION}

This is the first application of the SiD hybrid-less microstrip sensor, from which the sensor with its featured readout system
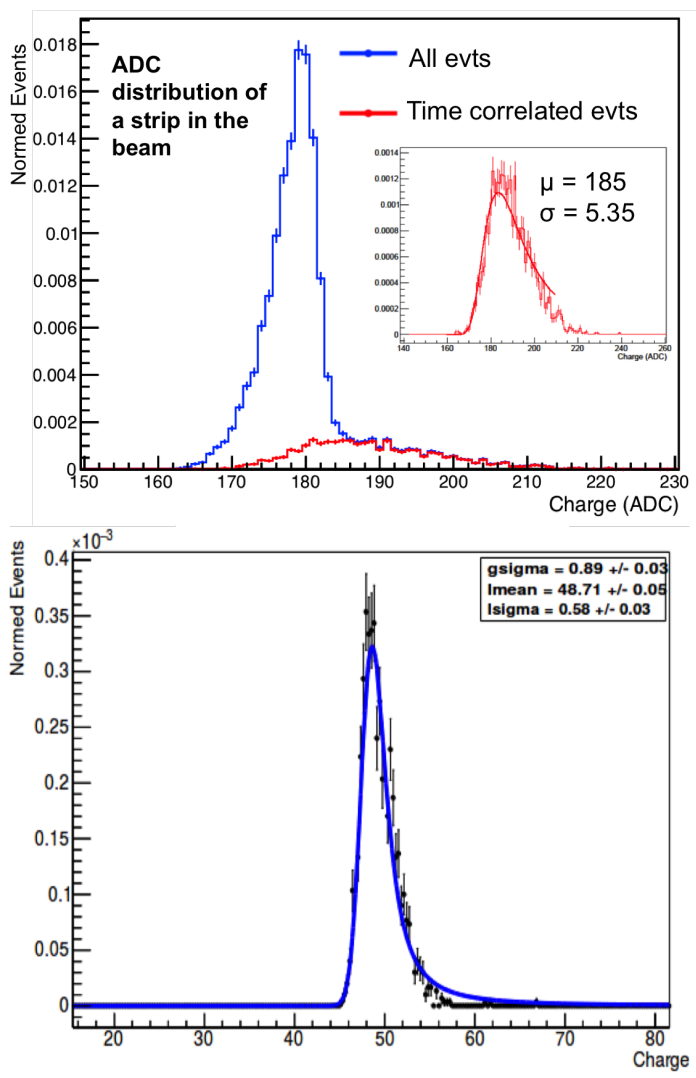

Fig. 10. ADC distribution of self-triggered events from one strip in the beam area. The upper figure includes a comparison plot from events matched external triggers in time (time correlated events) to all the self-triggered events, and a zoom-in view of the time correlated distribution with a promising Landau fit performed. The lower figure shows a different strip from a dataset with a tighter threshold, where the time correlated events overlapped with all the self-triggered events, and a good Landau convoluted Gaussian fit is performed.

are characterized systematically. The very first assembled modules were examined with various run conditions in the lab, and the following features are verified: 1) $80-85 \%$ readout channels shown good ADC to charge response, as well as relatively low noise $(\sim 0.5 \mathrm{fC}) ; 2)$ sensor is able to see signal out of noise under self-trigger mode, and locate the signal position.

The first beam test result not only confirms what has been verified in the lab, but also shows a lower noise $(\sim 0.2 \mathrm{fC})$ with the sensor better shielded with the final support structure. The external trigger run mode is first studied on this sensor at the test beam, and it turns out to give a promising signal to background ratio after pedestal subtraction. Signal efficiency is also first be tested under self-trigger mode with a timing coincidence to the external trigger,

\section{ACKNOWLEDGMENT}

The authors would like to thank Deutsches ElektronenSynchrotron DESY and Helmholtz Gesellschaft Funding for all the support. 


\section{REFERENCES}

[1] Advanced European Infrastructures for Detectors at Accelerators AIDA2020 Project Home Page, http://aida2020.web.cern.ch, Accessed on: Jan. 09, 2019.

[2] R. Diener et al., The DESY II test beam facility, arXiv:1807.09328v2 [physics.ins-det], Oct. 2018.

[3] D. Cussans, AIDA-2020 Deliverable Report 5.2: Triger Logic Unit ready, CERN, Geneva, AIDA-2020-D5.2, Dec. 2017.

[4] H. Jansen et al., Performance of the EUDET-type beam telescopes, EPJ Tech. Instrum., vol. 3, no. 1, pp. 7, Oct. 2016.

[5] J. Brau et al., KPiX - A 1,024 channel readout ASIC for the ILC, 2012 IEEE NSS/MIC, doi:10.1109/NSSMIC.2012.6551433.

[6] T. Behnke et al., The International Linear Collider Technical Design Report - Volume 4: Detectors, arXiv:1306.6329v1 [physics.ins-det], Jun. 2013.

[7] U. Kraemer et al., LYCORIS - A Large Area Strip Telescope, arXiv:1801.08505v1 [physics.ins-det], Jan. 2018. 